\title{
Mass transfer in eccentric binary systems using the binary evolution code BINSTAR
}

\author{
P. J. Davis, L. Siess, and R. Deschamps
}

\author{
Institut d'Astronomie et d'Astrophysique (IAA), Université Libre de Bruxelles, ULB, CP226, Boulevard du Triomphe, 1050 Brussels, \\ Belgium \\ e-mail: pdavis@ulb.ac.be
}

Received 17 September 2012 / Accepted 24 May 2013

\begin{abstract}
Context. Studies of interacting binary systems typically assume that tidal forces have circularized the orbit by the time Roche lobe overflow (RLOF) commences. However, recent observations of ellipsoidal variables have challenged this assumption.

Aims. We present the first calculations of mass transfer via RLOF for a binary system with a significant eccentricity using our new binary stellar evolution code. The study focuses on a $1.50+1.40 M_{\odot}$ main sequence binary with an eccentricity of 0.25 , and an orbital period of $P_{\text {orb }} \approx 0.7 \mathrm{~d}$. The reaction of the stellar components due to mass transfer is analysed, and the evolution of mass transfer during the periastron passage is compared to recent smooth particle hydrodynamics (SPH) simulations. The impact of asynchronism and non-zero eccentricity on the Roche lobe radius, and the effects of tidal and rotational deformation on the stars' structures, are also investigated.

Methods. Calculations were performed using the state-of-the-art binary evolution code BINSTAR, which calculates simultaneously the structure of the two stars and the evolution of the orbital parameters.

Results. The evolution of the mass transfer rate during an orbit has a Gaussian-like shape, with a maximum at periastron, in qualitative agreement with SPH simulations. The Roche lobe radius is modified by the donor star's spin and the orbital eccentricity. This has a significant impact on both the duration and the rate of mass transfer. We find that below some critical rotation rate, mass transfer never occurs, while above some threshold, mass is transferred over the entire orbit. Tidal and rotational deformation of the donor star causes it to become over-sized, enhancing the mass transfer rate further by up to about a factor of ten, leading to non-conservative mass transfer. The modulation of the mass transfer rate with orbital phase produces short-term variability in the surface luminosity and radius of each star. The longer-term behaviour shows, in accordance with studies of circular systems with radiative stars, that the donor becomes ever small and under-luminous, while the converse is the case for the accretor.
\end{abstract}

Key words. binaries: general - binaries: close - stars: evolution - methods: numerical

\section{Introduction}

Roche lobe overflow (RLOF) is of fundamental importance for a wide variety of binary star systems (for a review see Pringle 1985), such as cataclysmic variables, low-mass X-ray binaries and Algols. RLOF impacts upon both the binary orbit through the exchange (or perhaps the loss from the system) of mass and angular momentum, and on the evolution of the stellar components themselves, which may lead to a variety of exotic events. Accretion onto a high-mass white dwarf, for example, may lead to Type Ia supernovae (see Wang \& Han 2012, for a review), or novae events (see e.g. Warner 1995). Alternatively, a common envelope phase is expected to result from RLOF from a red or asymptotic giant branch star (Pazcynski 1976). Clearly, the outcome of binary evolution greatly depends upon both the rate at which material is transferred, and upon the nature of the stars.

Briefly, during RLOF, material from the donor (with mass $M_{1}$ ) is channelled as a narrow stream through the inner Lagrangian point, $\mathcal{L}_{1}$, which falls into the gravitational potential well of the accretor (mass $M_{2}$ ). Depending on the initial separation, the matter stream will either impact directly onto the surface of the companion star, or form an accretion disk around it. The mass transfer rate through the $\mathcal{L}_{1}$ point is determined by how much the star over-fills its Roche lobe, which in turn is dictated by the Roche lobe geometry and the donor's structure (see Ritter 1988; D’Antona et al. 1989).
The validity of the Roche model rests on three assumptions: that the stellar components can be treated as point masses, that the orbit is circular and that the donor star is rotating synchronously with the orbit. Studies of binaries undergoing RLOF generally consider that the last two of these assumptions hold by the time RLOF commences (e.g. Willems \& Kolb 2004), the reason being that tidal forces act on very short timescales, owing to the strong dependence of the tidal torques on the ratio of the stellar radius with the orbital separation (Zahn 1977). Therefore, tides will have circularized the orbit, and synchronized the donor star with the orbit, by the time RLOF starts.

However, the assumption of circular orbits for RLOF has been challenged by observations. Petrova \& Orlov (1999) compiled a catalogue of 128 eccentric binaries which reveals that approximately 15 per cent of these systems are semi-detached, while 5 per cent have evolved into contact. Nicholls \& Wood (2012) confirmed large eccentricities, between 0.14 and 0.42 , among seven ellipsoidal variables. This is a surprising result because these systems, which are close to filling their Roche lobes, should be nearly circular given their short separation and the efficiency of the tidal torques. These observations suggest that some of these binaries will therefore fill their Roche lobes while still possessing eccentric orbits. The idea of episodic mass transfer at periastron corroborates the hypothesis of Jorissen et al. (2009) to explain the evolution of KIII-type giants on the eccentricityorbital period plane. 
From a theoretical point of view, the assumption of a circular orbit with a synchronously rotating donor star during RLOF has also been called into question by Sepinsky et al. (2007b, 2009). They investigated the secular orbital evolution of an eccentric binary with a $1.44 M_{\odot}$ neutron star paired with a range of donor masses, as a result of both conservative and nonconservative mass transfer via RLOF at periastron. They compared the timescale for the evolution of the eccentricity due to tides and RLOF and found that, in contrast to tides which always act to circularize the orbit, mass transfer may either increase or decrease the eccentricity, over timescales ranging from a few Myr to a Hubble time. Furthermore, the timescale over which mass transfer acts to increase the eccentricity may be shorter than the tidal timescale which acts to decrease it. This occurs for mass ratios $q=M_{1} / M_{2} \lesssim 0.6$, which lies in the low-mass binary regime. Similar behaviour was also found by Sepinsky et al. (2010) who studied the orbital evolution of eccentric binaries by investigating the gravitational interaction between the matter stream and the stellar components. Hence, it is not guaranteed that mass transfer will circularize the orbit.

The eccentric nature of the binary orbit also means that the Roche model assumptions (i.e. where circular orbits and synchronous rotation is assumed) no longer applies (e.g. Limber 1963; Savonije 1978). Sepinsky et al. (2007a) analysed the effect of eccentricity and asynchronism on the Roche geometry. They found that the Roche lobe radius for a donor star which is rotating super-synchronously with the orbital motion at periastron will be smaller than the radius calculated using the classical Eggleton (1983) formula. The converse is true for a subsynchronously rotating donor star. As pointed out by Lajoie \& Sills (2011b, henceforth LS11) this will impact upon both the rate and duration of mass transfer.

The recent attempts to simulate mass transfer in eccentric binaries have all used SPH techniques (e.g. Regôs et al. 2005; Church et al. 2009; Lajoie \& Sills 2011a, LS11), and did not consider the possibility to address the problem using a binary stellar evolution code, which can accurately model the internal structure of the stellar components. To the best of our knowledge, only Edwards \& Pringle (1987) have attempted to calculate RLOF in eccentric binaries analytically. However, they considered a binary with a small eccentricity of $5 \times 10^{-4}$, and only modelled the flow in the vicinity of the $\mathcal{L}_{1}$ point.

In this paper, we present the first simulation of mass transfer for significantly eccentric systems using the state-of-the-art binary evolution code BINSTAR (Siess et al. 2013). To facilitate comparisons with the work by LS11, we consider their $1.50+1.40 M_{\odot}$ binary, with an eccentricity of $e=0.25$. In the light of the study by Sepinsky et al. (2007a) we investigate the affect of asynchronous rotation and eccentricity on the Roche lobe radius, and how this impacts on the mass transfer rate. We also examine the response of the structure of each star due to mass transfer, and to the deformation caused by rotation and tides. The secular orbital evolution of the binary system is deferred to a future study.

The paper is structured as follows. In Sect. 2, we describe the BINSTAR code, how we calculate the Roche lobe radii, the mass transfer rate, and how we account for the effects of rotation and tides on the stellar structure. Our results are presented in Sect. 3, which are discussed in Sect. 4. We conclude with a summary of our investigation in Sect. 5.

\section{Computational method}

BINSTAR is an extension of the single star evolution code STAREVOL. For further details regarding the stellar input physics, we refer the reader to Siess et al. (2000) and Siess (2006, 2007, 2009, 2010), and references therein.

In the following sections, we describe the key binary input physics used in this investigation. For a thorough description of the BINSTAR code, we refer the reader to Siess et al. (2013).

\subsection{Roche lobe radius}

In an eccentric orbit, the separation between the two stellar components, $D$, changes with time. For an orbit with a semi-major axis $a$ and an eccentricity $e, D$ is found from

$D=\frac{a\left(1-e^{2}\right)}{1+e \cos v}$

where $v$ is the true anomaly. Accordingly, the Roche lobe radius of the donor star, $R_{\mathcal{L}_{1}}$, will change along the orbit. The expression for $R_{\mathcal{L}_{1}}$ as given by Eggleton (1983) can be modified by replacing $a$ with $D$, i.e.

$R_{\mathcal{L}_{1}}=\frac{0.49 q^{2 / 3}}{0.6 q^{2 / 3}+\ln \left(1+q^{1 / 3}\right)} D$.

Henceforth, we term this the standard Roche lobe formalism.

However, this formula is strictly valid only for circular orbits and for donors, which are rotating synchronously with the orbit. We follow Sepinsky et al. (2007a) and calculate $R_{\mathcal{L}_{1}}$ by taking the eccentricity of the orbit and any asynchronism of the donor star into account. The potential in this case (normalized to the gravitational potential of the accretor, $\frac{G M_{2}}{D}$ ) is given by (Sepinsky et al. 2007a)

$$
\begin{aligned}
\Psi(x, y, z)= & -\frac{q}{\left(x^{2}+y^{2}+z^{2}\right)^{\frac{1}{2}}}-\frac{1}{\left[(x-1)^{2}+y^{2}+z^{2}\right]^{\frac{1}{2}}} \\
& -\frac{1}{2} \mathcal{A}(1+q)\left(x^{2}+y^{2}\right)+x,
\end{aligned}
$$

where the $x$-axis lies along the line joining the centres of mass of the two stars, in the direction from the donor to the accretor, the $z$-axis is perpendicular to the plane of the orbit and is parallel to the spin angular velocity of the donor, and the $y$-axis is perpendicular to the $x$-axis, and completes a right-handed coordinate set. All coordinates are given in units of $D$. In Eq. (3)

$\mathcal{A}=\frac{f^{2}(1+e)^{4}}{(1+e \cos v)^{3}}$

quantifies the degree of asynchronism and eccentricity, and $f$ is the spin angular speed of the donor star in units of the orbital angular speed at periastron, i.e.

$f=\frac{\Omega_{1}}{\omega_{\text {peri }}}$

Here, the orbital angular speed, $\omega$, is given by

$\omega=\frac{2 \pi}{P_{\text {orb }}} \frac{(1+e \cos v)^{2}}{\left(1-e^{2}\right)^{3 / 2}}$

where $P_{\text {orb }}$ is the orbital period. Since, in general, the potential in an eccentric system is varying with time, this will induce oscillations of mass elements inside the star, and perturb its hydrostatic equilibrium. However, Sepinsky et al. (2007a) show that such motions are negligible if the dynamical timescale, $\tau_{\text {dyn }}=\left(G M_{1} / R_{1}^{3}\right)^{-1 / 2}$ of the donor is much less than the tidal timescale, $\tau_{\text {tide }}=2 \pi /\left|\omega-\Omega_{1}\right|$, in which case the instantaneous 
shape of the star can be approximated by the instantaneous surfaces of constant $\Psi$ (e.g. Limber 1963; Savonije 1978). This so-called "first approximation" is valid for main sequence stars where $P_{\text {orb }} \gtrsim 10 \mathrm{~h}$ (Sepinsky et al. 2007a), hence for our $0.7 \mathrm{~d}$ system.

Sepinsky et al. (2007a) provide fit formulae for $R_{\mathcal{L}_{1}}$ as a function of $\mathcal{A}$ and $q$ for eccentric orbits and asynchronous donors. However, because of discontinuities in some regions of the $(\mathcal{A}, q)$ parameter space (Sepinsky, priv. comm.), the volume of the Roche lobe is calculated numerically using a 32-point Gaussian quadrature integration technique (Press et al. 1990), from which we derive the equivalent Roche lobe radius.

\subsection{Calculating mass transfer rates}

To calculate the mass transfer rates during each periastron passage, we follow the prescription outlined in Ritter (1988) and Kolb \& Ritter (1990).

We consider a donor star of mass $M_{1}$, radius $R_{1}$, effective temperature $T_{\text {eff, } 1}$, and with a mean molecular weight and density at the photosphere, $\mu_{\mathrm{ph}, 1}$ and $\rho_{\mathrm{ph}, 1}$, respectively. The mass transfer rate, $\dot{M}_{1}$, in the case where material is removed from the optically thin region of the donor's atmosphere (i.e. where the optical depth is $\tau \lesssim \frac{2}{3}$ ) is calculated using

$-\dot{M}_{1}=\dot{M}_{0} \exp \left(\frac{R_{1}-R_{\mathcal{L}_{1}}}{\hat{H}_{\mathrm{P}}}\right)$,

(Ritter 1988) where $\hat{H}_{\mathrm{P}}=H_{\mathrm{P}} / \gamma$ is the pressure scale height of the donor at the location of $\mathcal{L}_{1}$, which can be calculated from the pressure scale height at the photosphere, $H_{\mathrm{P}}$, and a correction factor, $\gamma$, which accounts for the geometry of the Roche lobe (see Appendix A). Also, $\dot{M}_{0}$ is the mass transfer rate if the donor star exactly fills its Roche lobe, and is given by

$\dot{M}_{0}=\frac{2 \pi}{\sqrt{e}} F(q) \frac{R_{\mathcal{L}_{1}}^{3}}{G M_{1}}\left(\frac{\mathcal{R} T_{\mathrm{eff}, 1}}{\mu_{\mathrm{ph}, 1}}\right)^{3 / 2} \rho_{\mathrm{ph}, 1}$.

Here, $\mathcal{R}$ is the ideal gas constant, $G$ is the gravitational constant and $F(q)$ is a function of $q$, and is determined from the area of the equipotential surface which intersects with the $\mathcal{L}_{1}$ point:

$F(q)=q \hat{\beta}^{-3}\{g(q)[g(q)-1-q]\}^{-1 / 2}$.

In turn, $\hat{\beta}=\frac{R_{\mathcal{L}_{1}}}{D}$, and $g(q)$ is given by

$g(q)=\frac{q}{x_{\mathcal{L}_{1}}^{3}}+\frac{1}{\left(1-x_{\mathcal{L}_{1}}\right)^{3}}$,

where $x_{\mathcal{L}_{1}}$ is the distance from the centre of mass of the donor star to the $\mathcal{L}_{1}$ point, in units of $D$. For the Roche model, $x_{\mathcal{L}_{1}}$ can be determined by numerically solving

$\frac{q}{x_{\mathcal{L}_{1}}^{2}}-\frac{1}{\left(x_{\mathcal{L}_{1}}-1\right)^{2}}-\mathcal{A} x_{\mathcal{L}_{1}}(1+q)+1=0$

(Sepinsky et al. 2007a), with $\mathcal{A}=1$ for synchronously rotating stars in circular orbits.

If the donor is significantly overflowing its Roche lobe, or if the donor's radius and Roche lobe radius are not evolving in step, then Eq. (7) is no longer valid. Instead, mass is also lost from the optically thick layers of the star (where $\tau \gtrsim 2 / 3$ ). The mass transfer rate in this case becomes (see also Deloye et al. 2007)

$$
\begin{aligned}
-\dot{M}_{1}= & \dot{M}_{0}+2 \pi F(q) \frac{R_{\mathcal{L}_{1}}^{3}}{G M_{1}} \\
& \times \int_{R_{\mathcal{L}_{1}}}^{R_{\mathrm{ph}}} \Gamma_{1}^{1 / 2}\left(\frac{2}{\Gamma_{1}+1}\right)^{\frac{\Gamma_{1}+1}{2\left(\Gamma_{1}-1\right)}} \frac{G M_{r}(P \rho)^{\frac{1}{2}}}{r^{2}} \mathrm{~d} r
\end{aligned}
$$

where $P, T, \mu$ and $M_{r}$ is the pressure, temperature, mean molecular weight and the mass of the donor star respectively at the radial coordinate $r$, and $\Gamma_{1}=(\mathrm{d} \ln P / \mathrm{d} \ln \rho)_{\mathrm{ad}}$ is the adiabatic exponent. The integral in Eq. (12) is evaluated numerically from the $\mathcal{L}_{1}$ point to the photosphere (subscript "ph").

If the donor star is rotating asynchronously with the orbit, and the eccentricity is non-zero, Eq. (9) is modified according to (see Appendix A)

$F^{*}(q, \mathcal{A})=q \hat{\beta}^{-3}\{g(q)[g(q)-\mathcal{A}-q \mathcal{A}]\}^{-\frac{1}{2}}$,

where the asterisk makes the distinction from Eq. (9).

\subsection{Initial model}

\subsubsection{The binary model}

To compare our results with the SPH simulations of LS11, we consider their $1.50+1.40 M_{\odot}$ main sequence binary system configuration. They construct their SPH models from the theoretical density profiles of a $1.50 M_{\odot}$ donor star with a $1.40 M_{\odot}$ accreting companion, calculated from their stellar evolution code, YREC (Guenther et al. 1992; see LS11 for further details).

Their stars have a metallicity of $Z=0.001$, and an age of approximately $1.3 \mathrm{Gyr}$, representative of binary systems populating old open clusters. They use a mixing length parameter $\alpha_{\mathrm{MLT}}=1.71$, with no convective overshooting or rotation (Sills, priv. comm.). With their input physics, we find that the donor and accretor have a radius of approximately 1.4 and $1.2 R_{\odot}$ respectively, in agreement with LS11. We also follow LS11 and synchronize the spin angular velocity of the stars to the orbital angular speed at apastron, yielding $f \approx 0.36$. The surface angular speed of the stellar components is then $\Omega_{1}=\Omega_{2} \approx 6 \times 10^{-5} \mathrm{~s}^{-1}$. For simplicity, we assume that the stars rotate as solid bodies, since the treatment of differential rotation is beyond the scope of the paper.

Pertinent to this study, we find that each star possesses a thin surface convective envelope, due to the first ionization of hydrogen. Their radial extents are relatively small (approximately $0.0014 R_{\odot}$ for the $1.50 M_{\odot}$ donor, and $0.002 R_{\odot}$ for the $1.40 M_{\odot}$ accretor), and their masses negligible, but they are crucial for understanding the response of the stellar structure to mass changes (see Sect. 3.2). Each star also has two additional convection zones $^{1}$ associated with the first and second ionization of helium, but they play a secondary role. Henceforth, we denote the zone associated with the ionization of hydrogen as the surface convection zone. The upper layers of this convective region are super-adiabatic and energy transport via convection becomes increasingly inefficient, until all the flux is transported purely by radiation in the atmosphere. To aid numerical stability, the number of shells in the donor and accretor are kept constant and are equal to 818 and 847 , respectively.

1 For the accretor, the convection zones due to ionized hydrogen and the first ionization of helium are merged into a single zone. 


\subsubsection{Integrating the orbit}

With an eccentricity, $e=0.25$ and a semi-major axis, $a=$ $4.80 R_{\odot}$, the orbital separation at periastron is $r_{\text {peri }}=3.60 R_{\odot}$, and the orbital period $P_{\text {orb }} \approx 0.7$ days.

To resolve numerically the orbital motion, we impose the evolutionary timestep $\Delta t$ not to exceed some fraction, $\mathcal{F}_{\text {orb }}$, of the time it takes for the stars to travel the circumference $2 \pi a$, given a value $v_{\text {orb }}$ of the orbital speed at that orbital phase i.e.

$\Delta t=\mathcal{F}_{\text {orb }}\left(\frac{2 \pi a}{v_{\text {orb }}}\right)$,

with

$v_{\text {orb }}=\sqrt{G\left(M_{1}+M_{2}\right)\left(\frac{2}{D}-\frac{1}{a}\right)}$.

For our simulations, we use $\mathcal{F}_{\text {orb }}=5 \times 10^{-3}$, which give us timesteps of approximately $10^{-6} \mathrm{yr}$.

\subsection{Treatment of mass loss and mass gain}

In the surface layers of the star, variations in the luminosity result from the release of gravo-thermal energy per unit time and per unit mass

$\varepsilon_{\text {grav }}=-T\left(\frac{\partial s}{\partial t}\right)_{m}$,

where $s$ is the specific entropy, and the subscript $m$ denotes that the derivative is evaluated at a fixed mass coordinate. In the situation where mass is lost or gained by the star, mass and time can no longer be considered as independent variables. Following Neo et al. (1977) we use a pseudo-Lagrangian variable, $\tilde{q}_{i}$, for each $\operatorname{star} i=1,2$, which is defined as

$\tilde{q}_{i}=\frac{m_{i}-M_{i}^{\prime}}{M_{i}(t)-M_{i}^{\prime}}$,

where $M_{i}^{\prime}$ is the mass coordinate above which mass is lost or gained, $m_{i}$ is a mass coordinate located at $m_{i} \geq M_{i}^{\prime}$ and $M_{i}(t)$ is the stellar mass at time $t$. In those layers affected by a change in mass, $\tilde{q}_{i}$ varies between 0 and 1 . For the donor, we take the value of $M_{1}^{\prime}$ to coincide with the location of the Roche lobe radius inside the star. We ensure that the time-step, $\Delta t$, is small enough such that $\left|\dot{M}_{1}\right| \Delta t<M_{1}-M_{1}^{\prime}$. For the accretor, mass is deposited uniformly above the mass coordinate corresponding to a fraction $f_{\text {accr }}$ of the accretor's mass. Hence, $M_{2}^{\prime}=f_{\text {accr }} M_{2}$, where we arbitrarily set $f_{\text {accr }}=0.9$. We find that the response of the accretor to mass gain is independent of the value of $f_{\text {accr }}$.

In this scheme, Eq. (16) can be re-cast as (see Neo et al. 1977; Fujimoto \& Iben 1989)

$$
\begin{aligned}
\varepsilon_{\text {grav }} & =-T\left(\frac{\partial s}{\partial t}\right)_{\tilde{q}_{i}}+T\left(\frac{\partial s}{\partial \ln \tilde{q}_{i}}\right)_{t} \frac{\partial \ln M_{i}}{\partial t}, \\
& =\varepsilon_{\text {grav }}^{(\text {nh) }}+\varepsilon_{\text {grav }}^{(\mathrm{h})}
\end{aligned}
$$

where the subscripts $\tilde{q}_{i}$ and $t$, indicate that the derivatives are to be evaluated at constant $\tilde{q}_{i}$ and $t$, respectively. The first and second terms on the right hand side of Eq. (18) correspond to the non-homologous, $\varepsilon_{\text {grav }}^{(\mathrm{nh})}$, and homologous, $\varepsilon_{\text {grav }}^{(\mathrm{h})}$, gravo-thermal energy generation rates, respectively.
The gravo-thermal luminosity, $L_{\text {grav }}$, is then the sum of the non-homologous, $L_{\text {grav }}^{(\mathrm{nh})}$, and homologous, $L_{\text {grav }}^{(\mathrm{h})}$, contributions given by

$$
\begin{aligned}
L_{\text {grav }} & =L_{\text {grav }}^{(\mathrm{nh})}+L_{\text {grav }}^{(\mathrm{h})} \\
& =\int_{M_{i}^{\prime}}^{M_{i}} \varepsilon_{\text {grav }}^{(\mathrm{nh})} \mathrm{d} m+\int_{M_{i}^{\prime}}^{M_{i}} \varepsilon_{\text {grav }}^{(\mathrm{h})} \mathrm{d} m .
\end{aligned}
$$

It is unclear what fraction, $0 \leq \alpha_{\text {acc }} \leq 1$, of the accretion luminosity, $L_{\mathrm{acc}}$, is imparted to the stellar layers, and what fraction is radiated away. For the case of direct-impact accretion Ulrich \& Burger (1976) argue that, due to the small fraction of the accretor's surface that is covered by the hot-spot, the energy dissipated from the shock region will have a negligible effect on the internal structure of the star. For disc accretion, it is uncertain what fraction of the luminosity emitted from the star-disc boundary layer is absorbed by the accretor (e.g. Siess et al. 1997). As shown in Sect. 3.3, however, accretion occurs via direct impact. Hence, following e.g. Kippenhahn \& Meyer-Hofmeister (1977), Tout et al. (1999), Braun \& Langer (1995), we assume that the accreted mass has the same specific entropy as the shell in which it is deposited.

For the models presented in Sects. 3.1 and 3.2, we also assume that mass transfer is fully conservative, i.e. $\beta=\left|\dot{M}_{2} / \dot{M}_{1}\right|=$ 1 . We investigate the possibility of relaxing this assumption further in Sect. 3.3.

\subsection{Effects of tides and rotation on the stellar structure}

To account for the deformation of the stellar structure caused by tidal and rotational forces, we implemented the prescription described by Landin et al. (2009), and Song et al. (2009). Their method employs the technique developed by Kippenhahn \& Thomas (1970, henceforth KT70), and improved by Endal \& Sofia (1978), to quantify the distortion of the star in a 1-dimensional stellar evolution code.

Consider an equipotential, $\Psi$, of surface $S_{\Psi}$ and volume $V_{\Psi}$, which encloses a mass $M_{\Psi}$. Following KT70, the stellar structure equations are modified by applying the correction factors $f_{\mathrm{P}}$ and $f_{\mathrm{T}}$, which are respectively given by

$f_{\mathrm{P}}=\frac{4 \pi r_{\Psi}^{4}}{G M_{\Psi}} \frac{1}{S_{\Psi}\left\langle g^{-1}\right\rangle}$,

and

$f_{\mathrm{T}}=\left(\frac{4 \pi r_{\Psi}^{2}}{S_{\Psi}}\right)^{2} \frac{1}{\langle g\rangle\left\langle g^{-1}\right\rangle}$,

where $r_{\Psi}$ is the radius of a sphere with volume $V_{\Psi}$. The effective gravities, averaged over $S_{\Psi}$, are given by

$\langle g\rangle=\frac{1}{S_{\Psi}} \int_{0}^{\pi} \int_{0}^{2 \pi} g(r, \theta, \phi) r^{2} \sin \theta \mathrm{d} \theta \mathrm{d} \phi$,

where the local gravity, $g(r, \theta, \phi)$, is obtained by differentiating the potential at the considered location, $P(r, \theta, \phi)$, i.e.

$g(r, \theta, \phi)=\left[\left(\frac{\partial \Psi}{\partial r}\right)^{2}+\left(\frac{1}{r} \frac{\partial \Psi}{\partial \theta}\right)^{2}+\left(\frac{1}{r \sin \theta} \frac{\partial \Psi}{\partial \phi}\right)^{2}\right]^{1 / 2}$.

In this expression, the potential consists of four contributions; the spherically symmetric part of the gravitational potential, $\Psi_{\text {grav }}$, the cylindrically symmetric potential due to rotation, $\Psi_{\text {rot }}$, 
the potential due to tides $\Psi_{\text {tide }}$ and the (non-symmetric) gravitational potential due to the distortion of the star resulting from rotation, $\Psi_{\text {dist,tide }}$ and tides $\Psi_{\text {dist,rot }}$, and can be written

$$
\begin{aligned}
\Psi(r, \theta, \phi)= & \Psi_{\text {grav }}+\Psi_{\text {rot }}+\Psi_{\text {tide }}+\Psi_{\text {dist,rot }}+\Psi_{\text {dist,tide }} \\
= & \frac{G M_{\Psi}}{r}+\frac{1}{2} \Omega_{1}^{2} r^{2} \sin ^{2} \theta+\frac{G M_{2}}{D}\left[1+\sum_{j=2}^{4}\left(\frac{r_{0}}{D}\right)^{j} \mathcal{P}_{j}(\lambda)\right] \\
& -\frac{4 \pi}{3 r^{3}} \mathcal{P}_{2}(\cos \theta) \mathcal{J}+4 \pi G M_{2} \sum_{j=2}^{4} \frac{\mathcal{P}_{j}(\lambda)}{(r D)^{j+1}} \mathcal{I}_{j},
\end{aligned}
$$

where $\lambda \equiv \sin \theta \cos \phi, r$ is the distance from the centre of the star to $P(r, \theta, \phi)$ and $\mathcal{P}_{j}$ is the $j$ th Legendre polynomial. The integrals $\mathcal{J}$ and $\mathcal{I}_{j}$ are respectively given by

$\mathcal{J}=\int_{0}^{r_{0}} \rho \frac{r_{0}^{\prime 7}}{M_{\Psi}} \frac{5+\eta_{2}}{2+\eta_{2}} \Omega_{1}^{2}\left(r_{0}^{\prime}\right) \mathrm{d} r_{0}^{\prime}$

and

$\mathcal{I}_{j}=\int_{0}^{r_{0}} \rho \frac{r_{0}^{\prime 2 j+3}}{M_{\Psi}} \frac{j+3+\eta_{j}}{j+\eta_{j}} \mathrm{~d} r_{0}^{\prime}$

for $j=2,3,4$ (Kopal 1959). In Eqs. (24) to (26), $r_{0}$ is the mean radius of the considered equipotential surface, and $\eta_{j}$ is determined numerically by solving the Radau equation

$r_{0} \frac{\mathrm{d} \eta_{j}}{\mathrm{~d} r_{0}}+6 \frac{\rho\left(r_{0}\right)}{\bar{\rho}\left(r_{0}\right)}\left(\eta_{j}+1\right)+\eta_{j}\left(\eta_{j}-1\right)=j(j+1)$,

using the boundary condition $\eta_{j}(0)=j-2$ (see Landin et al. 2009; and Song et al. 2009, for details). In Eq. (27), $\bar{\rho}\left(r_{0}\right)$ is the mean density at $r_{0}$.

Note that in their study, Song et al. (2009) only use the above prescription to the interior of the star, while an analytical approximation is applied at the surface (Song, priv. comm.). Furthermore, they neglect terms higher than second order (i.e. $j>2$ ). In doing so, they predict equal values for the effective gravity at the location facing the companion and on the opposite side (see their Fig. 2). In contrast, we find that tidal deformation at the surface closer to the companion is larger, giving a lower effective gravity here, because in our approach we take the higher-order terms into account.

\section{Results}

In the next section, we present the mass loss rate from the donor calculated using the standard Roche lobe formalism (Eq. (2)), and in Sect. 3.2 we analyse the structural response of the stellar components due to the mass exchange. The impact of tides and rotation on the structure of the so-called "distorted models" is also discussed. In Sect. 3.3, we examine to what extent this evolution remains conservative, while in Sect. 3.4 the effects of asynchronism and eccentricity on the Roche lobe radius and mass loss rate are investigated.

\subsection{Mass transfer rates}

As we can see from Fig. 1, RLOF commences just before periastron, and ceases just after, with a maximum mass transfer rate at closest approach of $\dot{M}_{1} \approx 1.3 \times 10^{-5} M_{\odot} \mathrm{yr}^{-1}$ for the nondistorted model. Moving towards periastron, the donor's Roche lobe radius shrinks as the separation between the two stars decreases (Eq. (2)). The amount that the donor star overfills its

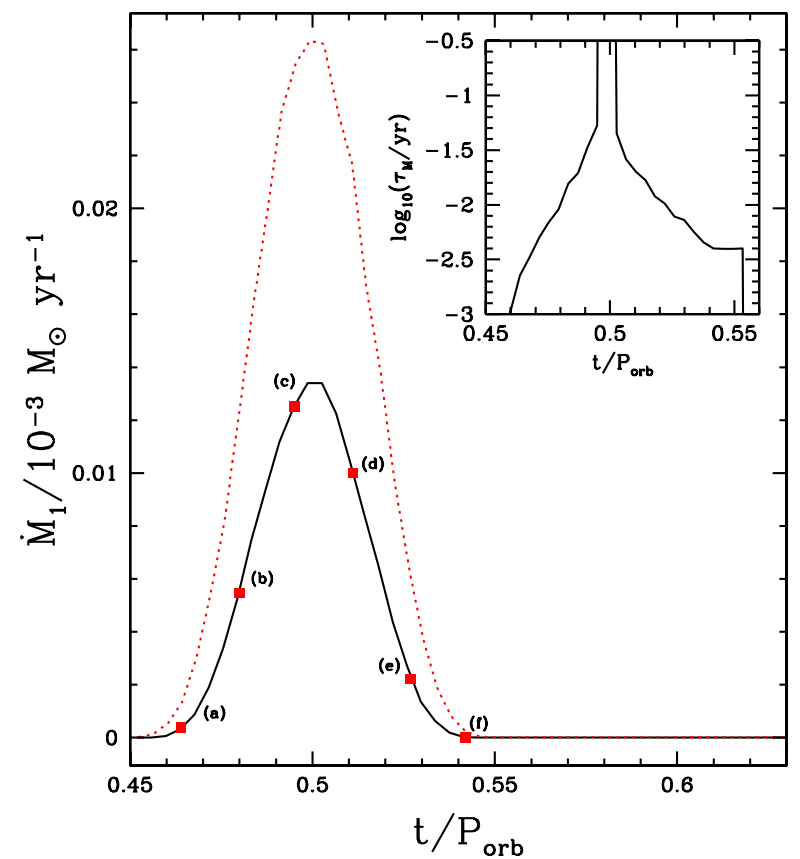

Fig. 1. Mass loss rate, $\dot{M}_{1}$, during the first periastron passage as a function of time since apastron, in units of $P_{\text {orb }}$ when the effects of tides and rotation on the donor's structure are ignored (black, solid curve) or included (red, dotted curve). The red squares labelled (a) to (f) on the black curve indicate different moments in time, and correspond to the panels labelled (a) to (f) in Figs. 2 and 6. The inset shows the evolution of the timescale over which the mass transfer rate changes, $\tau_{\dot{M}}=\left|\dot{M}_{1} / \ddot{M}_{1}\right|$ for the non-distorted model.

critical Roche surface (hereafter termed the overfilling factor) consequently rises, and $\dot{M}_{1}$ increases (Eqs. (7) and (12)). After reaching a maximum at periastron, both the degree of RLOF and $\dot{M}_{1}$ decline. This produces a Gaussian-like shape in the time evolution of $\dot{M}_{1}$, where the duration of RLOF is approximately 11 per cent of the orbital period.

For the distorted model, $\dot{M}_{1}$ (at a given phase) is higher than for the non-distorted case (dashed, red curve in Fig. 1), and at periastron peaks at approximately $2.6 \times 10^{-5} M_{\odot} \mathrm{yr}^{-1}$. The reason stems from the fact that the combined effects of tides and rotation reduce the effective surface gravity $\langle g\rangle$, increasing the donor's radius (a relative increase of about 0.4 per cent compared to the non-distorted model), and thus the overfilling factor at any orbital phase. The effect of increased stellar radius has also been reported within the SPH simulations of LS11 and Renvoizé et al. (2002; see also Uryu \& Eriguchi 1999, who use a different computational technique). The small increase in the donor's radius has a significant impact on $\dot{M}_{1}$ due to its sensitivity on the overfilling factor, but marginally affects the duration of mass transfer, increasing it to about 12 per cent.

Such a modulation of the mass transfer rate with orbital phase was suggested to account for a change in the speed of the bipolar outflows emanating from the accretor of HD 44179 within the Red Rectangle. In this paradigm, the velocity of the ejected material is maximum at periastron, and minimum at apastron (Witt et al. 2009). Furthermore, variations of the X-ray luminosity of the intermediate-mass black hole HLX-1 (Lasota et al. 2011) and of the optical and ultra-violet luminosity of the symbiotic system BX Mon (Leibowitz et al. 2011) have also been attributed to such a modulation of the mass transfer rate. 

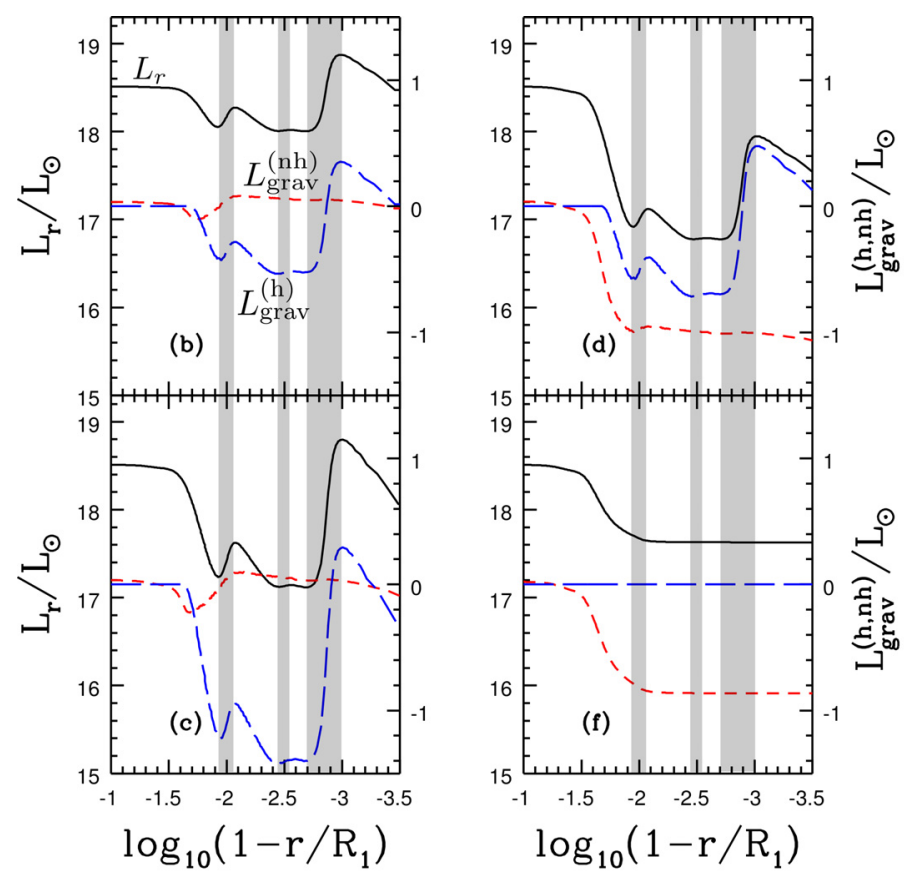

Fig. 2. Stellar luminosity profile, $L_{r}$ (black solid curve, left axis), and contributions from the homologous, $L_{\text {grav }}^{\text {(h) }}$ (blue, short-dashed curve, right axis) and the non-homologous, $L_{\mathrm{grav}}^{(\mathrm{nh})}$ (red, long-dashed curve, right axis) terms for the non-distorted donor star at different moments in time, specified in the bottom left-hand corner of each panel (see Fig. 1). The shaded regions indicate convection zones.

\subsection{Reaction of the stars}

In contrast to circular orbits, mass transfer occurs periodically during each periastron passage, and changes with the orbital phase (Fig. 1), which gives rise to a corresponding short-term variability of the radius and luminosity of each star. The response of the donor and accretor during mass transfer is discussed in Sects. 3.2.1 and 3.2.2, respectively.

\subsubsection{The donor star}

In accordance with e.g. Webbink (1977a) and Neo et al. (1977), the action of removing mass from radiative layers (where the entropy gradient is positive) absorbs energy $\left(\partial L_{\text {grav }}^{(\mathrm{h})} / \partial r<\right.$ 0 ; see blue, short-dashed curves in Fig. 2). Conversely, in the super-adiabatic region (right-most shaded area in Fig. 2) mass loss causes a slight increase in $L_{r}$ because in those layers $\partial L_{\text {grav }}^{(\mathrm{h})} / \partial r>0$.

As discussed in Webbink (1976), a changing mass transfer rate will perturb the thermal structure of the star over a timescale $\tau_{\dot{M}}=\left|\dot{M}_{1} / \ddot{M}_{1}\right|$. Initially, $\tau_{\dot{M}}$ is so short (inset of Fig. 1) that only the outermost surface layers, which consist of the surface convection zone, can restore thermal equilibrium on a timescale $\tau_{\text {th }} \ll \tau_{\dot{M}}$. In these super-adiabatic layers (which encompass about 3 per cent of the donor radius at point (b)), $\epsilon_{\text {grav }}^{(\mathrm{nh})} \approx 0$ and the luminosity increases due to the dominant contribution from $\epsilon_{\text {grav }}^{\text {(h) }}$ (panel (b), Fig. 2).

Subsequently, as $\dot{M}_{1}$ increases towards periastron, an evergrowing deficit in $L_{\text {grav }}^{(\mathrm{h})}\left(\propto \dot{M}_{1}\right)$ within the radiative layers develops. In parallel, as $\tau_{\dot{M}}$ is also increasing, the condition $\tau_{\text {th }}<\tau_{\dot{M}}$ moves deeper (encompassing about 10 per cent of the donor's radius at point (c)). Because $L_{\text {grav }}^{(\mathrm{h})}$ dominates over a larger fraction of the radiative envelope, the luminosity deficit grows in these layers (black curves in Fig. 2) and entails an over-all reduction of the star's surface luminosity $L_{1}$ (e.g. point (c), left panel of Fig. 3) and radius $R_{1}$ (left panel, point (c) on black curve of Fig. 4), as attested by $L_{\text {grav }}^{(\mathrm{nh})}>0$ (red long-dashed curves, panels (b) and (c) in Fig. 2).

Away from periastron, both $\left|L_{\text {grav }}^{(\mathrm{h})}\right|$ and $\tau_{\dot{M}}$ decline. The former will still cause $R_{1}$ and $L_{1}$ to shrink (Figs. 3 and 4, e.g. point (d)) but at a slower rate. The latter will give rise to a decrease in $L_{\text {grav }}^{(\mathrm{nh})}$ due to decompression of the deep radiative layers where $\tau_{\dot{M}}<\tau_{\text {th }}$, and eventually $L_{\text {grav }}^{(\mathrm{nh})}$ becomes negative (panel (d) of Fig. 2). Once mass loss shuts off, the radiative layers expand as energy flows from the interior to fill the luminosity deficit and restore thermal equilibrium (Fig. 2, panel (f)), causing a corresponding rise in $L_{1}$ and $R_{1}$ (e.g. point (f) in Figs. 3 and 4).

By the time the donor starts overfilling its Roche lobe at the second periastron passage, the star has not yet fully recovered thermal equilibrium and $R_{1}$ and $L_{1}$ are slightly smaller than at the beginning of the simulation. Subsequent mass transfer episodes perturb the structure further, and $R_{1}$ and $L_{1}$ become ever smaller at each new mass transfer episode (black curve, Fig. 5). Despite the short-term reaction of the donor during periastron, the aforementioned longer-term behaviour is in accordance with studies of predominantly radiative stars in circular orbits (e.g. Webbink 1977a,b; Siess et al. 2013).

For the distorted model (red, dotted curves in Figs. 3 and 4), the donor is over-sized and under-luminous compared to the non-distorted model for all orbital phases. The evolution of $R_{1}$ and $L_{1}$ during the periastron passage are qualitatively the same as for the non-distorted model, but for two main differences. Firstly, before mass transfer starts, $R_{1}$ rises while $L_{1}$ declines. This is because, as the stellar separation shrinks, the surface gravity decreases due to the strengthening of the tidal interaction terms on the right hand side of Eq. (24). In turn, the stars' structure will be less compact, and the reverse process occurs once the stellar separation increases. Secondly, the evolution of $R_{1}$ and $L_{1}$ during mass transfer is perturbed more significantly, due to the larger mass transfer rates obtained in the tidally distorted case, as discussed in Sect. 3.1.

\subsubsection{The accreting star}

As with the donor, the reaction of the accretor is initially dictated by the surface convection zone, but because the extent of that region is about twice that of the donor's, it governs the reaction over a longer duration (almost until periastron, see panels (b) and (c) in Fig. $6^{2}$ ). The response of the accretor is opposite to that of the donor star; mass addition to the convective layers causes the surface luminosity, $L_{2}$, and radius, $R_{2}$, to shrink (points (b) to (c) in right panels of Figs. 3 and 4).

As the stars move away from periastron, the compression decelerates as $\tau_{\dot{M}}$ declines, and $L_{\text {grav }}^{(\mathrm{nh})}$ increases. The positive contribution of $L_{\text {grav }}^{(\mathrm{h})}$ due to the response of the radiative layers dominates (panel (d), Fig. 6), causing a rise in $L_{2}$ and an expansion of the surface layers (e.g. point (d), Figs. 3 and 4).

At $t \geq 0.53 P_{\text {orb }}$ since apastron, mass accretion has become negligible $\left(L_{\text {grav }}^{(\mathrm{h})} \approx 0\right)$ and contraction resumes $\left(L_{\text {grav }}^{(\mathrm{nh})}>0\right.$; see panel (f), Fig. 6) as the excess energy originally created in the radiative layers by the deposition of matter is now radiated away.

\footnotetext{
2 The values of $L_{\text {grav }}^{(\mathrm{nh})}$ and $L_{\mathrm{grav}}^{(\mathrm{h})}$ may seem high compared to the surface luminosity but what is physically relevant is the sum of these two contributions which, as previously stated, is not affected by the choice of $f_{\text {accr. }}$.
} 
P. J. Davis et al.: Mass transfer in eccentric binaries
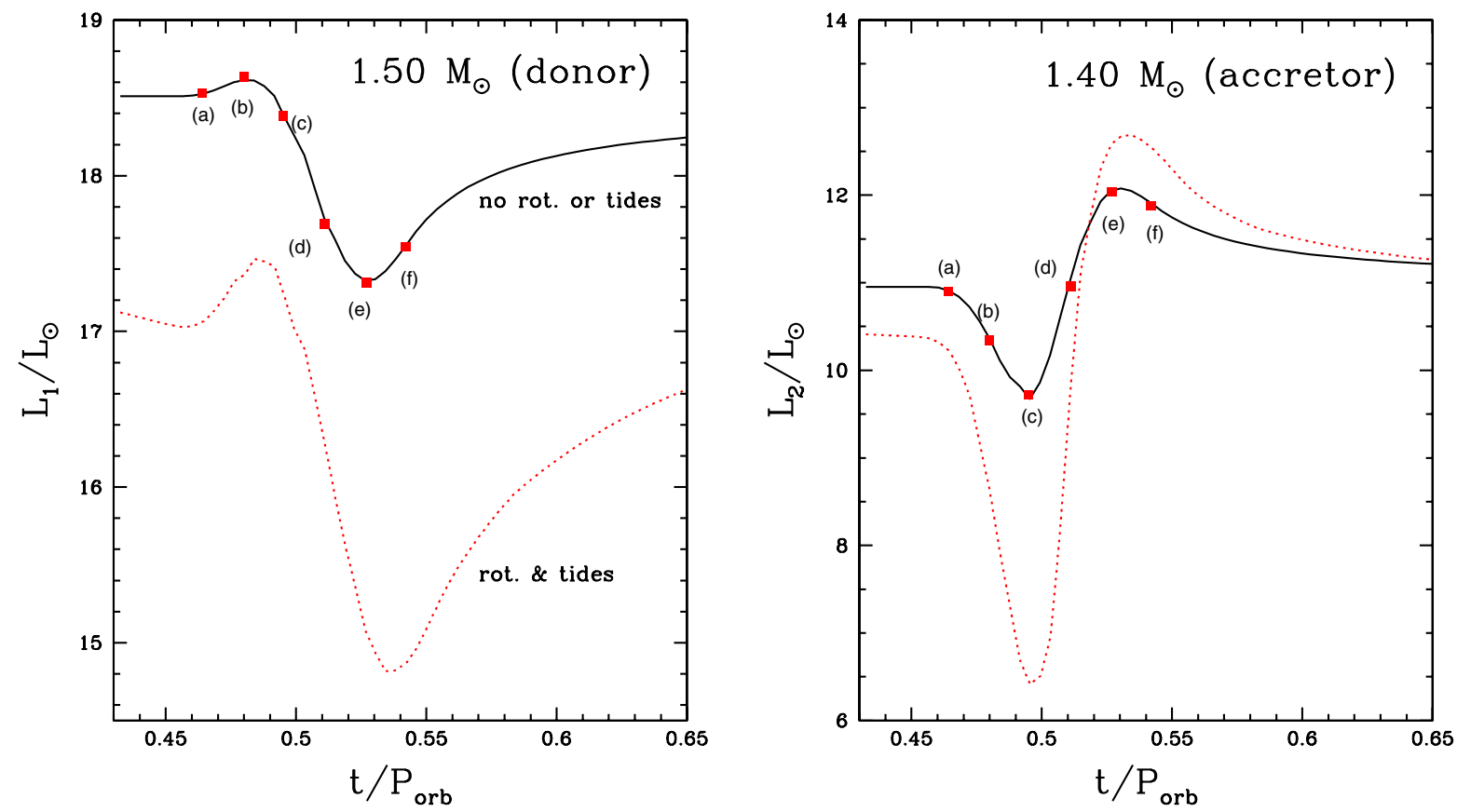

Fig. 3. Evolution of the surface luminosity for the donor, $L_{1}$ (left panel), and of the accretor, $L_{2}$ (right panel) during mass transfer at the first periastron passage. The meaning of the lines are the same as for Fig. 1. Points labelled (a) to (f) correspond to the points labelled (a) to (f) in Fig. 1.
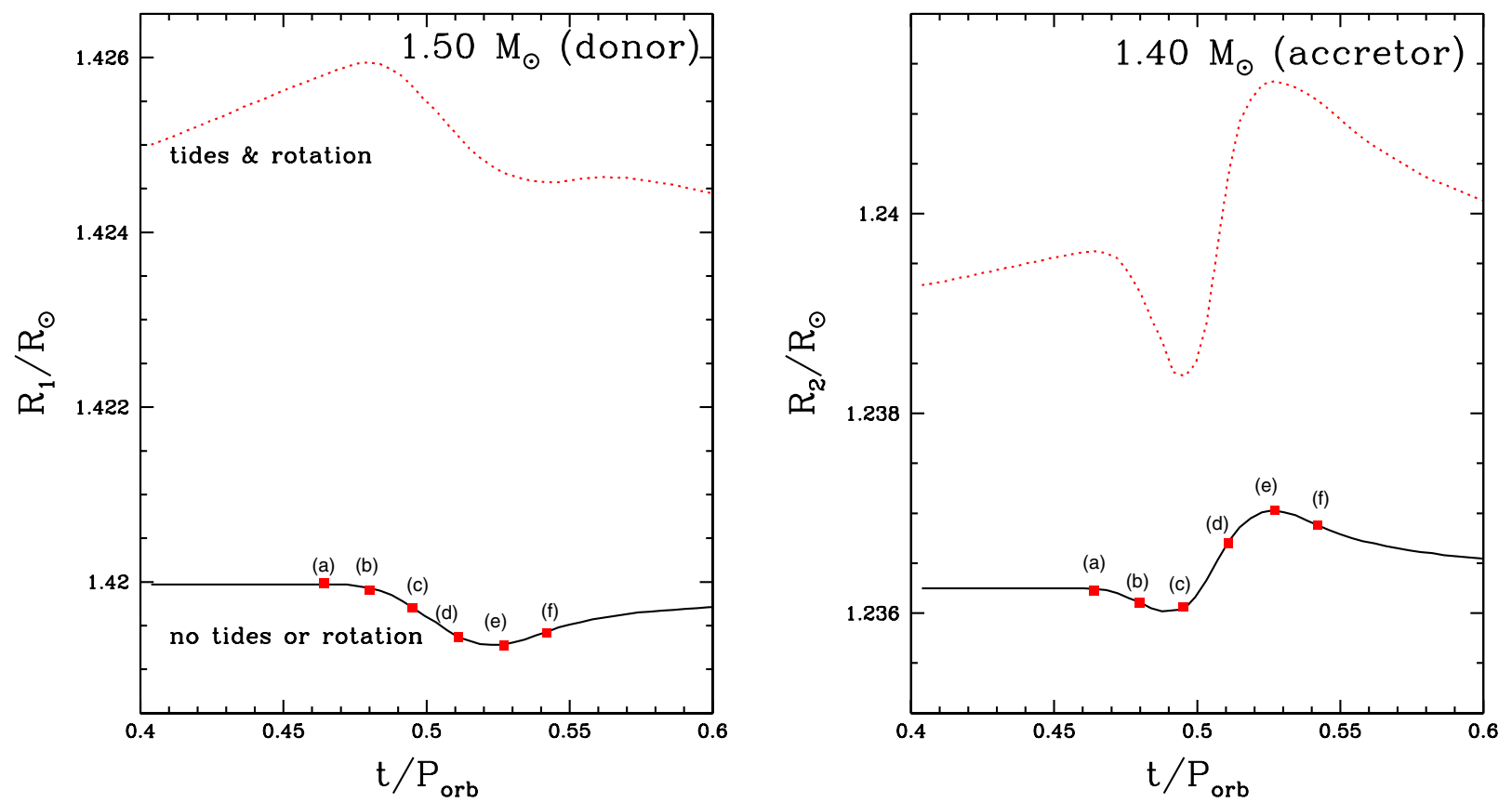

Fig. 4. Similar to Fig. 3, but now depicting the evolution of the radius for the donor $R_{1}$ (left panel) and the accretor $R_{2}$ (right panel) during the first periastron passage.

Note that $\left|L_{\text {grav }}^{(\mathrm{nh})}\right|$ is much smaller (panel (f)) because the accretor is no longer perturbed by mass deposition, and is relaxing towards thermal equilibrium. Hence, $L_{2}$ and $R_{2}$ decrease slightly (point (f) in Figs. 3 and 4). Subsequent mass transfer episodes causes $L_{2}$ and $R_{2}$ to gradually rise from orbit to orbit (red, dotted curve in Fig. 5), again in accordance with studies of accreting radiative stars in circular binaries (e.g. Neo et al. 1977; Webbink 1976).

The impact of tides and rotation on the accretor is the same as for the donor, except that at $t \gtrsim 0.52 P_{\text {orb }}$, the distorted model has a somewhat higher luminosity than the non-distorted case. This is because the higher mass accretion rate creates a larger energy excess in the radiative layers below the surface convection zone due to compression.

For the non-distorted models, we find that, during the periastron passage, the surface luminosity of the donor decreases by as much as $\Delta L_{1} \approx-1.3 L_{\odot}$, while for the accretor it increases by as much as $\Delta L_{2} \approx 2 L_{\odot}$. However, these variations almost cancel out, and the net change in the luminosity for the entire system $\left(\Delta L_{\Sigma}=\Delta L_{1}+\Delta L_{2}\right)$ is $\Delta L_{\Sigma} \approx 0.7 L_{\odot}$. This corresponds to a variation of about 0.03 magnitude, which may not be easily observable. For the distorted models, on the other hand, we find $\Delta L_{1} \approx-2.6 L_{\odot}$ and $\Delta L_{2} \approx 6.5$, and so a change of about $0.1 \mathrm{mag}\left(\Delta L_{\Sigma} \approx 3.9 L_{\odot}\right)$, which detection may be more feasible. 


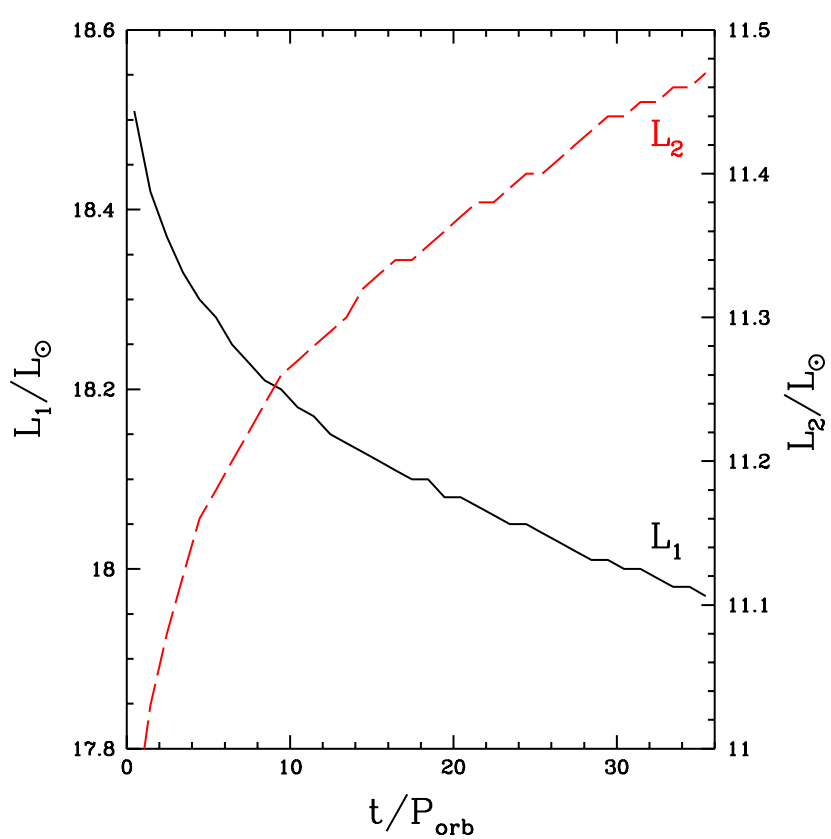

Fig. 5. Evolution of the surface luminosity of the donor $L_{1}$ (solid black curve, left axis) and of the accretor $L_{2}$ (dashed red curve, right axis) just before mass transfer recommences over a duration of about 30 periastron passages.

We emphasize, however, that despite the low values of $\Delta L_{\Sigma}$, the change in the intrinsic luminosities of each star is significant and takes place over a brief period of time (approximately $1-2 \mathrm{~h}$ in our model).

\subsection{Effect of direct impact accretion}

For an accretion disc to form, the minimum distance of approach between the matter stream and the accretor must satisfy

$R_{\min } \approx 0.0425 D[q(1+q)]^{1 / 4}>R_{2}$,

(Ulrich \& Burger 1976). For our system, $R_{\min } \approx 0.2 R_{\odot}<R_{2}$, implying direct impact accretion and the formation of a hot-spot, in accord with LS11. The presence of a hot-spot may give rise to mass ejection from the system. To investigate this possibility, we follow van Rensbergen et al. (2008), and briefly summarise below their formalism that we implemented within BINSTAR.

According to these authors, the hot-spot luminosity, $L_{\mathrm{HS}}$, is given by

$L_{\mathrm{HS}}=\frac{\alpha_{\mathrm{acc}}}{S_{\mathrm{acc}}} L_{\mathrm{acc}}=K L_{\mathrm{acc}}$,

where $S_{\text {acc }}$ is the fraction of the accretor's surface inhabited by the hot-spot and $\alpha_{\text {acc }}$ is the accretion efficiency. A fit to the variable $K$ was performed by van Rensbergen et al. (2011) using an observed sample of 13 Algol binaries, and is given by

$K \approx 3.9188\left(\frac{M_{1}+M_{2}}{M_{\odot}}\right)^{1.645}$.

The accretion luminosity, $L_{\mathrm{acc}}$, is calculated from the potential difference between the $\mathcal{L}_{1}$ point, $\Psi\left(x_{\mathcal{L}_{1}}, 0,0\right)$ and the point of impact, $\Psi_{\text {imp }}\left(x_{\text {imp }}, y_{\text {imp }}, 0\right)$ (using Eq. (3)), yielding

$L_{\text {acc }}=\left|\dot{M}_{1}\right|\left(\Psi_{\mathcal{L}_{1}}-\Psi_{\text {imp }}\right)$.

The point of impact, $\left(x_{\text {imp }}, y_{\text {imp }}\right)$, can be found from ballistic trajectory calculations. However, to do this for eccentric systems
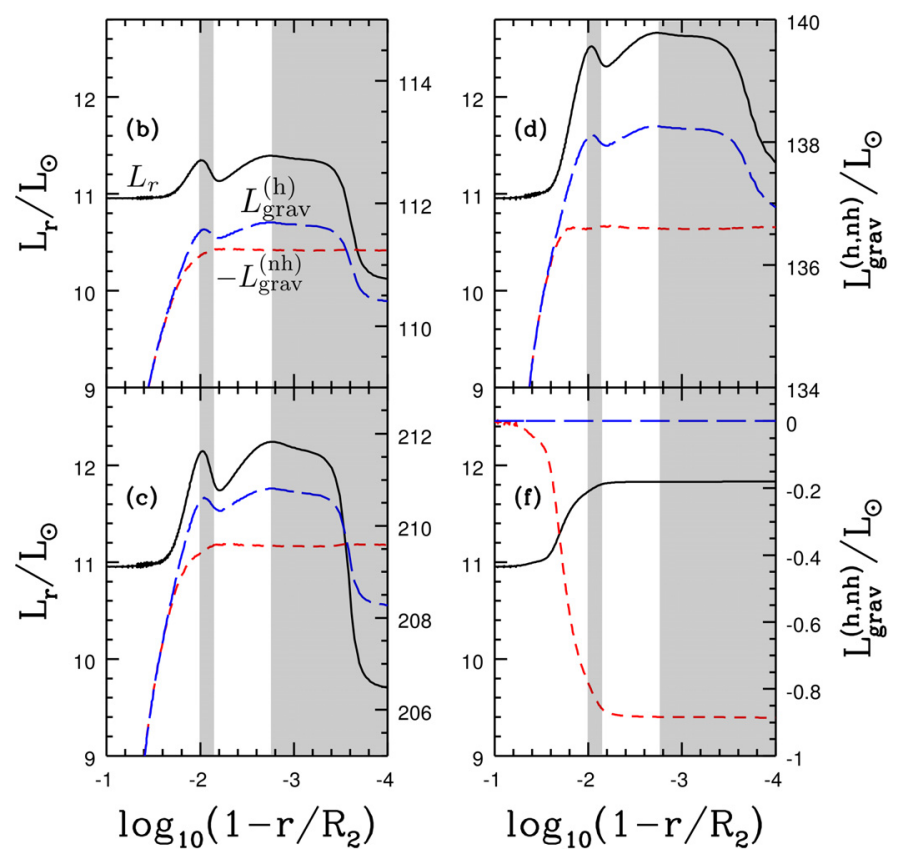

Fig. 6. Similar to Fig. 2 but now for the accretor. Note that $-L_{\text {grav }}^{(\mathrm{nh})}$ has been plotted (right axis).

where the separation, hence the potential, are varying with time, is beyond the scope of the paper. To estimate $\left(x_{\text {imp }}, y_{\text {imp }}\right)$, we use the angle between the point of impact and the line joining the two stars, $\varpi$, determined from the ballistic trajectories calculated by Flannery (1975) for different values of $q$ in circular orbits. For our system, we use $\varpi \approx 70^{\circ}$. From Eqs. (29) and (31), it is obvious that $L_{\mathrm{HS}}$ follows the same orbital modulation as $\dot{M}_{1}$, and if $\dot{M}_{1}$ exceeds some critical value $\dot{M}_{\text {crit }}$, the luminosity in the hotspot region may exceed the Eddington value, $L_{\text {Edd }}$, allowing for mass ejection. van Rensbergen et al. (2008) give

$$
\begin{aligned}
\dot{M}_{\text {crit }}= & \frac{1}{\Psi_{\mathcal{L}_{1}}-\Psi_{\text {imp }}}\left\{\left[\frac { L _ { \mathrm { Edd } } R _ { 2 } } { G M _ { 2 } } \left(\frac{G M_{1}}{r_{1, \text { imp }}}+\frac{G M_{2}}{R_{2}}+\frac{1}{2} r_{\mathrm{C}, \mathrm{imp}}^{2} \omega^{2}\right.\right.\right. \\
& \left.\left.\left.-\frac{1}{2} R_{2}^{2} \Omega_{2}^{2}\right)-L_{2}\right] \frac{1}{K}+\dot{E}_{\mathrm{rot}}\right\},
\end{aligned}
$$

where $r_{1, \text { imp }}$ and $r_{\mathrm{C} \text {,imp }}$ are the distances from the point of impact to the centre of mass of the donor and the binary system respectively and $\dot{E}_{\text {rot }}$ is the rate of change of the accretor's rotational kinetic energy. Due to the small amount of mass that is transferred (up to approximately $10^{-7} M_{\odot}$ in one orbit), the change in the accretor's spin speed is negligible over the timescale of the simulation, and $\dot{E}_{\mathrm{rot}} \approx 0$.

The temperature of the hot-spot, $T_{\mathrm{HS}}$, can be estimated from $\sigma T_{\mathrm{HS}}^{4}=L_{\mathrm{HS}} / S_{\mathrm{HS}}$, where $S_{\mathrm{HS}} \approx 10^{20} \mathrm{~cm}^{2}$ is the area of the hot-spot on our accretor's surface (see Gunn et al. 1999; van Rensbergen et al. 2008). At periastron, $T_{\mathrm{HS}} \approx 10^{5} \mathrm{~K}$, so the material in that region is fully ionized, justifying our use of the electron scattering opacity in the expression for $L_{\text {Edd }}$.

\subsection{Effects of asynchronism and eccentricity}

Figure 7 shows the effects on $\dot{M}_{1}$ of an eccentric orbit and an asynchronously rotating donor on the Roche lobe radius ${ }^{3}$

3 The step-like features near periastron, particularly evident for the synchronous and super-synchronous models, are a result of low spatial resolution in the surface layers of the donor star, revealed by the small time-steps used. This produces the corresponding features in Fig. 9. 


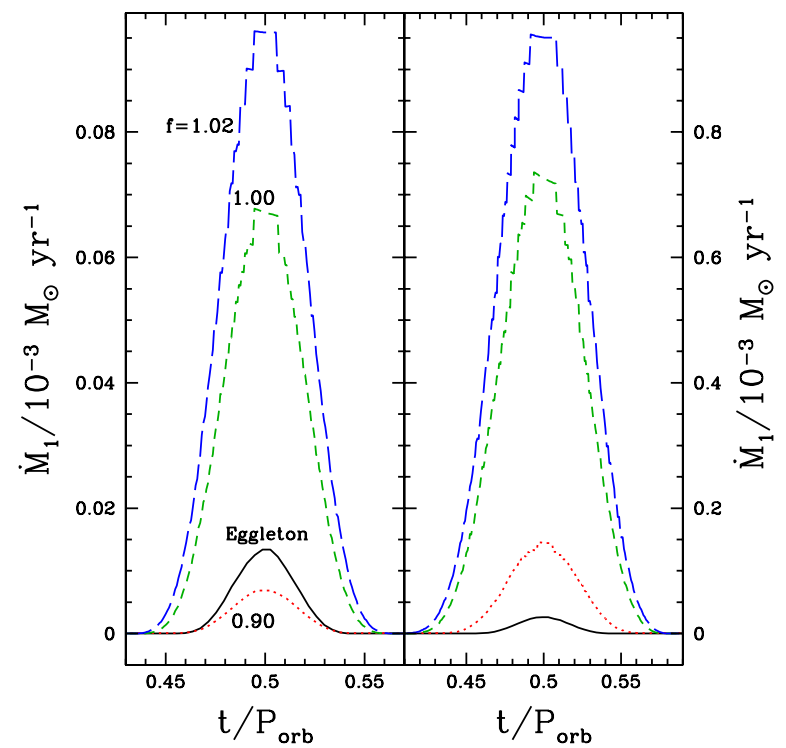

Fig. 7. Left panel, left axis: similar to Fig. 1, but here the effects of asynchronism and eccentricity on the Roche lobe radius are taken into account (Eqs. (3) and (4)). Black, solid curve: standard Roche lobe formalism; red, dotted curve: $f=0.90$; green, short-dashed curve: $f=1.00$; blue, long-dashed curve: $f=1.02$. Right panel, right axis: the same but for the distorted models.

(Eqs. (3) and (4)). Three cases are considered: sub-synchronous rotation of the donor with the orbital motion at periastron, $f=$ 0.90 (red, dotted curves), synchronous rotation ( $f=1.00$, green, short-dashed curves) and super-synchronous rotation $(f=1.02$, blue, long-dashed curves).

For the non-distorted models, at a given orbital phase, increasing the value of $f$ increases $\dot{M}_{1}$. At periastron, for example, $\dot{M}_{1}$ rises from approximately $7.0 \times 10^{-6} M_{\odot} \mathrm{yr}^{-1}$ for the sub-synchronous case to about $9.6 \times 10^{-5} M_{\odot} \mathrm{yr}^{-1}$ for the supersynchronous case; about a factor of 14 increase. The explanation resides in the fact that a higher value of $f$ yields a smaller Roche lobe radius. In turn, the donor will over-fill its Roche lobe further (i.e. an increase in $\left(R_{1}-R_{\mathcal{L}_{1}}\right) / R_{1}$ with increasing $f$; see top panel of Fig. 8), which will cause the mass transfer rate to grow (Eqs. (7) and (12)). Despite the enhanced mass loss rate, we find that mass transfer is still fully conservative. Values of $\dot{M}_{\text {crit }}$ are higher than $\dot{M}_{1}$ at periastron by about a factor of 100 for the sub-synchronous case to about a factor of 6 for the supersynchronous case.

On the other hand, for the tidally and rotationally distorted models, the mass transfer rate at periastron is typically a factor of 10 higher than for the non-distorted configuration, for a given value of $f$. This increase in $\dot{M}_{1}$ leads to both a higher hot-spot luminosity (as high as about $10^{5} L_{\odot}$ at periastron for the distorted, super-synchronous case) and non-conservative evolution for the synchronous and super-synchronous models (top panel, Fig. 9), with values of $\dot{M}_{\text {crit }}$ of $7 \times 10^{-4} M_{\odot} \mathrm{yr}^{-1}$ and $6 \times 10^{-4} M_{\odot} \mathrm{yr}^{-1}$, respectively. The accretion efficiency $\beta$ at a given phase is smaller for the super-synchronous case, because for this model $\dot{M}_{1}$ is higher, and so it further exceeds $\dot{M}_{\text {crit }}$ leading to more mass ejection. Due to the modulation of $\dot{M}_{1}$ with orbital phase, $\beta$ is a minimum at periastron. During one orbit, the total ejected mass from the accretor is about $10^{-9} M_{\odot}$ and $2 \times 10^{-8} M_{\odot}$ for the synchronous and super-synchronous models, respectively (bottom panel, Fig. 9).

The change in the Roche lobe radius with $f$ can be explained in terms of the location of the $\mathcal{L}_{1}$ point. Recall that $\mathcal{L}_{1}$ is where $\partial \Psi / \partial x=0$, i.e. where a particle experiences no net

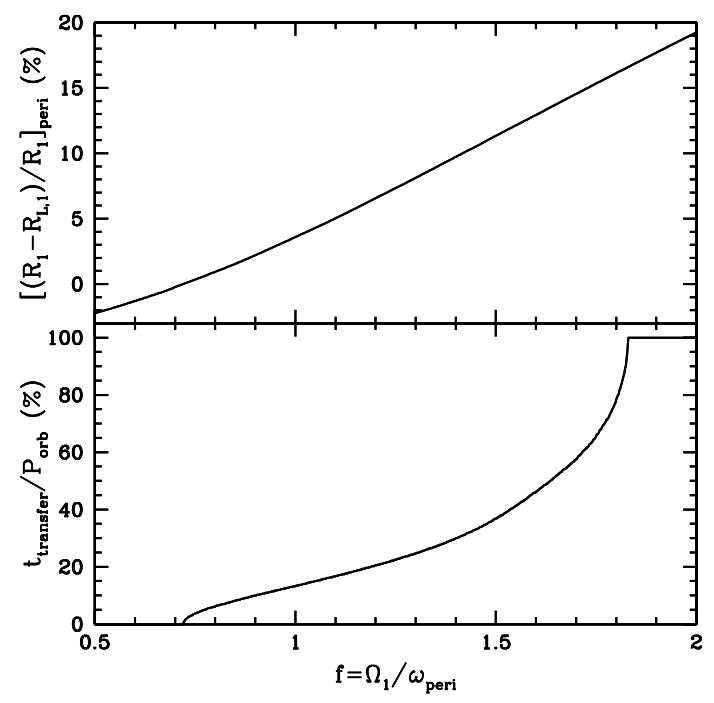

Fig. 8. Relative difference between the donor radius and its Roche lobe radius at periastron, $\left[\left(R_{1}-R_{\mathcal{L}_{1}}\right) / R_{1}\right]_{\text {peri }}$ (top panel), and the duration of mass transfer, $t_{\text {transfer }}$, in units of the orbital period (bottom panel), as a function of $f$ for our $1.50 M_{\odot}$ donor.

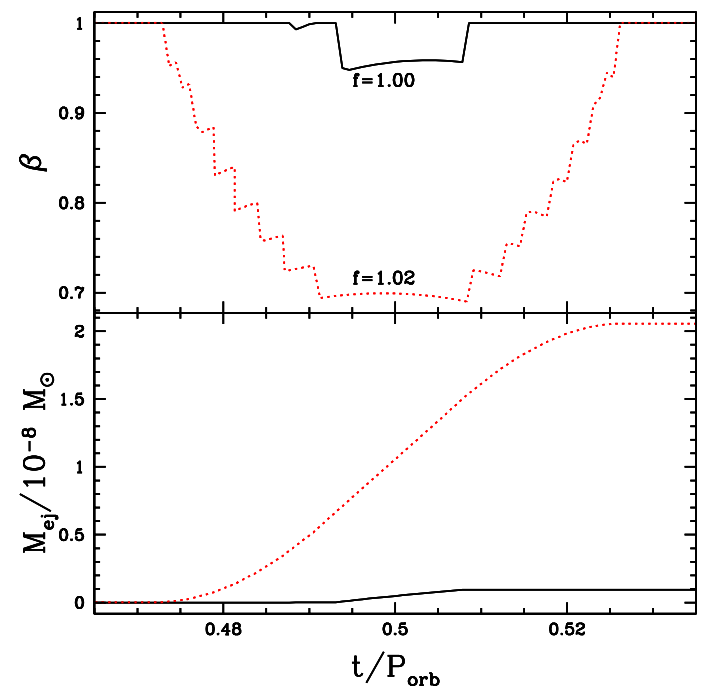

Fig. 9. Top panel: accretion efficiency, $\beta=\left|\dot{M}_{2} / \dot{M}_{1}\right|$ versus time for the distorted models, where $R_{\mathcal{L}_{1}}$ is determined from Eqs. (3) and (4), with $f=1.00$ (black, solid curve) and $f=1.02$ (red, dotted curve). Bottom panel: mass of ejected material, $M_{\mathrm{ej}}$ (in units of $10^{-8} M_{\odot}$ ), as a function of time.

acceleration, due to the balance of the centrifugal and gravitational accelerations. Let the position of the $\mathcal{L}_{1}$ point at a given phase for a given value of $f$ be $x_{\mathcal{L}_{1}}(0)$. If $f$ is then increased (causing a corresponding increase in $\mathcal{A}$ ), then a test particle at $x_{\mathcal{L}_{1}}(0)$ will experience a stronger centrifugal acceleration, hence an outward displacement. The new location of $x_{\mathcal{L}_{1}}$ therefore needs to be situated closer to the donor star $\left[x_{\mathcal{L}_{1}}<x_{\mathcal{L}_{1}}(0)\right]$ to re-establish a net zero-acceleration. Since the Roche equipotential surface passes through the $\mathcal{L}_{1}$ point, a decreasing value of $x_{\mathcal{L}_{1}}$ means that both the volume and the radius of the Roche lobe will shrink.

A shrinking Roche lobe radius with rising $f$ also means that mass transfer will occur for a longer duration, because mass transfer will start (end) earlier (later) in the orbit. This duration, $t_{\text {transfer }}$, is shown in the bottom panel of Fig. 8. For our super-synchronous case, mass transfer lasts for approximately 17 per cent of the orbital period. 
We also see that if $f \lesssim 0.7$, mass transfer does not occur at all. This is because the Roche lobe radius of the supersynchronously rotating donor increases so much, compared to the value obtained from Eq. (2), that the donor star never fills it. Indeed, as Fig. 8 shows, $\left(R_{1}-R_{\mathcal{L}_{1}}\right) / R_{1}<0$ at periastron for $f \lesssim 0.7$. Therefore, if we evolve our $1.50+1.40 M_{\odot}$ system with $f=0.36$, our system will never undergo mass transfer, in contrast with LS11. At periastron, for $f=0.36$, the relative increase in the Roche lobe radius due to asynchronous rotation, compared to the value obtained from Eq. (2), is approximately 6 per cent, in agreement with Sepinsky et al. (2007a). We return to the comparison of our work with that of LS11 in Sect. 4. On the other hand, for $f \gtrsim 1.8$, Fig. 8 shows that mass transfer will occur over the whole orbit, since at all phases $R_{\mathcal{L}_{1}}<R_{1}$.

Finally, the change in $\mathcal{A}$ with orbital phase, will cause a corresponding change in the donor's Roche lobe radius. To understand this more clearly, we can recast Eq. (4) in terms of the instantaneous ratio of the spin angular speed of the donor star, and the orbital angular velocity, i.e.

$\mathcal{A}=\left(\frac{\Omega_{1}}{\omega}\right)^{2}(1+e \cos v)$.

As we move from periastron to apastron, the orbital speed decreases. Therefore, for a given value of $\Omega_{1}$, the donor star will rotate progressively more super-synchronously with the orbital motion, causing a corresponding rise in both $\mathcal{A}$, and the centrifugal acceleration. Consequently, the Roche lobe radius will be progressively smaller than the value calculated by the standard Eggleton (1983) formalism, as apastron is approached.

\section{Discussion}

The evolution of the mass transfer rate has a Gaussian-like behaviour, with a maximum value occurring at periastron, in excellent agreement with the findings of LS11. However, we also report major differences.

LS11 obtain a maximum mass transfer rate which occurs in fact slightly after periastron. This delay is due to the time required by the material ejected from the donor to fall down the potential well of the companion star and be accreted. This is approximately the free-fall time, of the order of $\tau_{\mathrm{ff}} \approx 0.06 P_{\text {orb }}$ (LS11). In BINSTAR, this delay is absent because the code does not currently follow the ballistic trajectory of the ejected material. Instead, it assumes that mass transfer occurs instantaneously. The delay found by LS11 is more physical as a result of their more realistic treatment of mass transfer, but it does not change the overall picture.

Furthermore, we emphasize that our determination of the point of impact on the accretor's surface based on the angle $\varpi$ is rather crude. Sepinsky et al. (2010) calculated ballistic trajectories in eccentric binaries for a single particle ejected at periastron. They found that the particle may fall back onto the donor star, as well as fall onto the accretor via a disc or direct impact. However, as discussed below, mass transfer occurs over a significant fraction of the orbital period, and not just at periastron. Furthermore, their study neglects the contribution of the thermal sound speed at the $\mathcal{L}_{1}$ point to the ejected particle's velocity. It is unclear how these modifications would impact on the ballistic trajectory and on the mass transfer.

If we take the effects of asynchronous rotation of the donor (with $f \approx 0.36$ ) and the eccentricity of the orbit on the Roche lobe radius into account, our donor never fills its Roche lobe, even if the effect of tides, which act to increase the donor radius, are considered. This is in contrast to LS11, who find that mass transfer lasts for approximately 25 per cent of the orbital period, and peaks at periastron at a value of $\dot{M}_{1} \approx 2 \times 10^{-3} M_{\odot} \mathrm{yr}^{-1}$. In their simulation, tidal and rotational forces increase the radius of their donor to a greater extent, to approximately $1.8 R_{\odot}$, so that the donor is sufficiently large to fill its Roche lobe near periastron. As a check, we re-ran a simulation using a slightly more evolved donor star with $R_{1} \approx 1.8 R_{\odot}$, again accounting for the effects of asynchronism and eccentricity on the value of $R_{\mathcal{L}_{1}}$. Now, we find that mass transfer lasts for about 30 per cent of the orbital period, in good agreement with LS11, but our peak mass transfer rate is approximately a factor of 35 larger than theirs. This may arise from the uncertainty in $R_{1}$, since LS11 cannot provide a precise value. Even though this uncertainty is small, it may greatly impact upon $\dot{M}_{1}$ due to the sensitivity on the degree of overflow (Eqs. (7) and (12)).

The technique employed by LS11 to determine mass transfer rates is obviously more physically realistic than the 1-dimensional analytical scheme used in the present study. The assumptions underlying Eqs. (7) and (12) that the flow is laminar, and that material moves along the equipotential surfaces is not strictly valid. Instead, material flows upwards from within the Roche lobe of the donor, rather than along its surface (Eggleton 2006), which would have the effect of increasing the mass transfer rate. On the other hand, the pressure gradients will produce turbulence in the flow rather than bulk motion of the material, and will contribute to reduce the mass transfer rate. Ge et al. (2010), who use a similar formalism to Eq. (12), estimate that the calculated mass transfer rate is accurate to within about a factor of 2 .

Despite the fact that LS11 distribute their SPH particles according to density profiles calculated from their stellar evolution code, they do not consider the transport of energy due to convection and radiation, (similarly to SPH simulations by Regós et al. 2005; and Church et al. 2009). Instead, LS11 apply a simple polytropic equation of state of the form

$P=\rho(\gamma-1) u$,

where $u$ is the specific internal energy of the stellar material, and $\gamma=5 / 3$. While a value of $\gamma=5 / 3$ is a reasonable approximation for the deep optically thick layers of the interior, it is inadequate to treat the regions of partial ionization or small optical thickness. Considering that the layers of the star in LS11 are adiabatic, their SPH simulations will over-estimate the pressure and the temperature in these layers, yielding larger radii. This 'relaxed' SPH stellar configuration will be able to expand to a greater extent due to tidal and rotational deformation, and impact on the mass transfer rate.

Even though our simulations are able to provide a more realistic internal structure, the treatment of tides and rotation outlined in Sect. 2.5 remains an approximation, since all 3-dimensional effects are folded into a 1-dimensional formalism (see also Knigge et al. 2011, for a discussion of this matter). It is therefore unclear whether the discrepancy in stellar radii, and therefore the mass transfer rate, is due to the polytropic approximation used by LS11 or the subtleties of the KT70 method used here.

For our distorted, super-synchronous model, which gives a similar mass transfer rate at periastron as found by LS11, mass transfer is non-conservative. Over one orbit, the total mass lost by the donor is approximately $1 \times 10^{-7} M_{\odot}$, while the total accreted mass is about $8 \times 10^{-8} M_{\odot}$, i.e. 20 per cent of the transferred material is ejected by the accretor. However, in contrast to SPH simulations, we cannot follow the ultimate fate of 
this material. For their $1.50+1.40 M_{\odot}$ system, 20 per cent of the transferred material formed an envelope around the system, while 5 per cent was ejected. On the other hand, since LS11 do not model the thermal structure of the hot-spot, they may underestimate the fraction of material that is ejected from the system.

It is evident from Figs. 7 and 8 that the degree of asynchronism of the donor star has a significant impact on both the mass loss rate and the duration of mass transfer. It should be pointed out that the mass transfer rates predicted by the standard Eggleton (1983) formalism (which assumes that the star is rotating synchronously with the orbit at each location) and the $f=1.00$ case with non-zero eccentricity differ by approximately a factor of about 5 for the non-distorted models. This factor is further enhanced to about 60 due to tidal and rotational effects on the donor star. Hence, it is imperative that the effect of eccentricity and asynchronism on the Roche lobe geometry, and the distortion of the donor star, be accounted for.

In the Sepinsky et al. (2007b, 2009) studies, the structure and the response of the donor star were not taken into account, and mass transfer was modelled as a delta function at periastron. This is in contrast with Lajoie \& Sills (2011a), LS11 and the present work which show that the episode of mass exchange represents a non-negligible fraction of the orbital period. Allowing for non-instantaneous mass transfer may impact on the evolution of the binary system in a way that is different to the delta function model.

\section{Summary}

This study paves the first steps towards calculating mass transfer rates for significantly eccentric binaries, taking the effects of tides and rotation on the structures of the stellar components into account, as well as the effects of eccentricity and asychronous rotation of the donor on the Roche lobe radius. In this paper, we have used a state-of-the-art binary evolution code BINSTAR to calculate mass transfer rates for a $1.50+1.40 M_{\odot}$ main sequence binary system, with an eccentricity of 0.25 and an orbital period of $P_{\text {orb }} \approx 0.7 \mathrm{~d}$ and we compared our results with the SPH calculations performed by LS11 for the same system.

The evolution of the mass transfer rate with time shows Gaussian-like behaviour, with a maximum mass transfer rate occurring at periastron, in qualitative agreement with LS11. The accretion luminosity (which emanates from a hotspot for this particular system) also varies in response to the changing mass transfer rate. The duration of the mass transfer rate represents a non-negligible fraction of the orbital period, particularly if the donor star is rotating sufficiently rapidly. On the other hand, mass transfer may not occur at all if the donor is rotating too slowly.

During RLOF, the timescale over which the mass transfer rate changes, $\tau_{\dot{M}}=\left|\dot{M}_{1} / \ddot{M}_{1}\right|$ is so short that only the outer-most stellar layers, consisting of the surface convection zone, can restore thermal equilibrium. Initially, the response of each star is dictated by this convection zone. As mass transfer proceeds, a larger fraction of the stars' envelope, consisting of the radiative layers, dictate the subsequent response of each star.

The evolution of the luminosity of the donor and accretor over 30 orbits (Fig. 5) highlights the fact that the longer-term evolution of the stellar structure is what we would expect for stars with significantly radiative envelopes, as shown by studies of such stars in circular orbits.

Finally, tidal and rotational forces increase the surface radius of the donor, enhancing mass transfer further by a factor of about 10 , potentially leading to non-conservative mass transfer.
In the future, we wish to follow the mass transfer rate as a function of orbital phase over many orbits, and determine the secular orbital evolution of eccentric binaries.

Acknowledgements. P.J.D. acknowledges financial support from the Communauté Française de Belgique - Actions de recherche Concertées, from the Université Libre de Bruxelles and from an FNRS Fellowship. P.J.D. would also like to thank U. Kolb and J. Sepinsky for many useful discussions, and A. Sills for providing further details of the stellar models used in the SPH simulations. We also thank S. Toupin for his assistance with the development of the BINSTAR routines which calculates the Roche lobe radius, and the distortion of the star due to tides and rotation. Finally, we are grateful to the anonymous referee whose constructive comments improved the quality of the manuscript. L.S. is a FNRS research associate.

\section{Appendix A: Determining the Function $F^{*}(q, \mathcal{A})$}

In the following description, we use a coordinate system where the origin lies at the centre of mass of the donor. The positive $x$-axis points from the centre of mass of the donor to the centre of mass of the accretor, while the $z$-axis is perpendicular to the orbital plane, and is parallel to the donor's spin angular velocity. The $y$-axis lies on the orbital plane, and forms a right-handed set.

We start with Eq. (20) of Sepinsky et al. (2007a), which gives the potential, $\Psi$ (normalized by the potential due to the accretor, $\left.\frac{G M_{2}}{D}\right)$

$$
\begin{aligned}
\Psi= & -\frac{q}{\left(x^{2}+y^{2}+z^{2}\right)^{\frac{1}{2}}}-\frac{1}{\left[(x-1)^{2}+y^{2}+z^{2}\right]^{\frac{1}{2}}} \\
& -\frac{1}{2} \mathcal{A}(1+q)\left(x^{2}+y^{2}\right)+x,
\end{aligned}
$$

where all coordinates are given in units of the instantaneous separation, $D$ and $\mathcal{A}$ is given by Eq. (4). Following Meyer \& Meyer-Hofmeister (1983) and Ritter (1988), we can express the change in the potential around the vicinity of the $\mathcal{L}_{1}$ point, $\Delta \Psi$, using a Taylor expansion to the second order, according to

$\Delta \Psi \approx B y^{2}+C z^{2}$,

where

$B=\frac{1}{2}\left(\frac{\partial^{2} \Psi}{\partial y^{2}}\right)_{\mathcal{L}_{1}}=\frac{1}{2}[g(q)-\mathcal{A}-q \mathcal{A}]$,

where the subscript $\mathcal{L}_{1}$ indicates that the derivative is evaluated at the inner-Lagrangian point, and $g(q)$ is given by Eq. (10). Similarly,

$C=\frac{1}{2}\left(\frac{\partial^{2} \Psi}{\partial z^{2}}\right)_{\mathcal{L}_{1}}=\frac{1}{2} g(q)$.

Recalling that the potential has been expressed in units of the secondary's gravitational potential, and that the distances are in units of the separation, then converting back to 'normal' units gives us for $B$ and $C$, respectively,

$$
\begin{aligned}
B & =\frac{G M_{2}}{2 D^{3}}[g(q)-\mathcal{A}-q \mathcal{A}] \\
& =\frac{G M_{1}}{2 q D^{3}}[g(q)-\mathcal{A}-q \mathcal{A}], \\
C & =\frac{G M_{1}}{2 q D^{3}} g(q) .
\end{aligned}
$$

We can see from Eq. (A.2) that the equipotential surface in the plane of the $\mathcal{L}_{1}$ point is an ellipse with an area, $S$, given by

$$
S=\pi \frac{\Delta \Psi}{\sqrt{B C}} \text {. }
$$


Following Meyer \& Meyer-Hofmeister (1983), the area of the cross section of the flow, $Q$, is then

$Q=\pi \frac{\mathcal{R} T_{\mathrm{eff}, 1}}{\mu_{\mathrm{ph}, 1}} \frac{1}{\sqrt{B C}}=\frac{2 \pi \mathcal{R} T_{\mathrm{eff}, 1}}{\mu_{\mathrm{ph}, 1}} \frac{D^{3} q}{G M_{1}}\{g(q)[g(q)-\mathcal{A}-q \mathcal{A}]\}^{-\frac{1}{2}}$.

Defining

$\hat{\beta}=\frac{R_{\mathcal{L}_{1}}}{D}$,

then substituting Eq. (A.9) into (A.8) gives us

$Q=\frac{2 \pi \mathcal{R} T_{\mathrm{eff}, 1}}{\mu_{\mathrm{ph}, 1}} \frac{R_{\mathcal{L}_{1}}^{3}}{G M_{1}} q \hat{\beta}^{-3}\{g(q)[g(q)-\mathcal{A}-q \mathcal{A}]\}^{-\frac{1}{2}}$.

The mass transfer rate when the donor star exactly fills its Roche lobe, $\dot{M}_{0}$, is given by (Ritter 1988)

$\dot{M}_{0}=\frac{1}{\sqrt{e}} \rho_{\mathrm{ph}, 1} v_{\mathrm{s}} Q$,

where

$v_{\mathrm{s}}=\frac{\mathcal{R} T_{\mathrm{eff}, 1}}{\mu_{\mathrm{ph}, 1}}$

is the sound velocity of the material in the location of the $\mathcal{L}_{1}$ point. Substituting Eqs. (A.10) and (A.12) into (A.11) and comparing the result with Eq. (8) gives us

$$
\begin{aligned}
\dot{M}_{0}= & \frac{2 \pi}{\sqrt{e}} q \hat{\beta}^{-3}\{g(q)[g(q)-\mathcal{A} q-\mathcal{A}]\}^{-1 / 2} \\
& \times \frac{R_{\mathcal{L}_{1}}^{3}}{G M_{1}}\left(\frac{\mathcal{R} T_{\mathrm{eff}, 1}}{\mu_{\mathrm{ph}, 1}}\right)^{3 / 2} \rho_{\mathrm{ph}, 1}, \\
= & \frac{2 \pi}{\sqrt{e}} F^{\star}(\mathcal{A}, q) \frac{R_{\mathcal{L}_{1}}}{G M_{1}}\left(\frac{\mathcal{R} T_{\mathrm{eff}, 1}}{\mu_{\mathrm{ph}, 1}}\right)^{3 / 2} \rho_{\mathrm{ph}, 1},
\end{aligned}
$$

where

$$
F^{*}(A, q)=q \hat{\beta}^{-3}\{g(q)[g(q)-\mathcal{A} q-\mathcal{A}]\}^{-1 / 2},
$$

as given by Eq. (13).

Finally, the pressure scale scale height at the location of the $\mathcal{L}_{1}$ point is calculated using

$\hat{H}_{\mathrm{P}}=\frac{H_{\mathrm{P}}}{\gamma(q)}$

where $\gamma(q)$ takes the non-spherical shape of the donor's Roche lobe into account, and is given by (Ritter 1988)

$\gamma(q)= \begin{cases}0.954+0.025 \log _{10} q-0.038\left(\log _{10} q\right)^{2} & \text { if } 0.04 \leq q<1 \\ 0.954+0.039 \log _{10} q-0.114\left(\log _{10} q\right)^{2} & \text { if } 1 \leq q<20\end{cases}$

\section{References}

Braun, H., \& Langer, N. 1995, A\&A, 297, 483

Church, R. P., Dischler, J., Davies, M. B., et al. 2009, MNRAS, 395, 1127

D’Antona, F., Mazzitelli, I., \& Ritter, H. 1989, A\&A, 225, 391
Deloye, C. J., Taam, R. E., Winisdoeffer, C., \& Chabrier, G. 2007, MNRAS, 381, 525

Edwards, D. A., \& Pringle, J. E. 1987, MNRAS, 229, 383

Eggleton, P. P. 1983, ApJ, 268, 368

Eggleton, P. P. 2006, Evolutionary Processes in Binary and Multiple Stars, ed. P. Eggleton (Cambridge, UK: Cambridge University Press)

Endal, A. S., \& Sofia, S. 1976, ApJ, 210, 184

Flannery, B. P. 1975, MNRAS, 170, 325

Fujimoto, M. Y., \& Iben, I. Jr. 1989, ApJ, 341, 306

Ge, H., Hjellming, M. S., Webbink, R. F., Chen, X., \& Han, Z. 2010, ApJ, 717, 724

Guenther, D. B., Demarque, P., Kim, Y.-C., \& Pinsonneault, M. H. 1992, ApJ, 387,372

Gunn, A. G., Brady, P. A., Migenes, V., Spencer, R. E., \& Doyle, J. G. 1999, MNRAS, 304, 611

Jorissen, A., Frankowski, A., Famaey, B., \& Van Eck, S. 2009, A\&A, 498, 489

Kippenhahn, R., \& Meyer-Hofmeister, E. 1977, A\&A, 54, 539

Kippenhahn, R., \& Thomas, H.-C. 1970, in Stellar Rotation, ed. A. Slettebak (Holland: D. Reidel Publ. Co. Dordrecht), 20 (KT90)

Knigge, C., Baraffe, I., \& Patterson, J. 2011, ApJS, 194, 28

Kolb, U., \& Ritter H. 1990, A\&A, 236, 385

Kopal, Z. 1959, in Close Binary Systems (London, UK: Chapman \& Hall)

Lajoie, C.-P., \& Sills, A. 2011a, ApJ, 726, 66

Lajoie, C.-P., \& Sills, A. 2011b, ApJ, 726, 67, LS11

Landin, N. R., Mendes, L. T. S., \& Vaz, L. P. R. 2009, A\&A, 494, 209

Lasota, J.-P., Alexander, T., Dubus, G., et al. 2011, ApJ, 735, 89

Leibowitz, E. M., \& Formiggini, L. 2011, MNRAS, 414, 2406

Limber, D. N. 1963, ApJ, 138, 1112

Neo, S., Miyaji, S., Nomoto, K., \& Sugimoto, D. 1977, PASJ, 29, 249

Nicholls, C. P., \& Wood, P. R. 2012, MNRAS, 421, 2616

Paczyński, B. 1976, in Structure and Evolution of Close Binary Systems, eds. P. Eggleton, S. Mitton, \& J. Whelan, IAU Symp., 73, 75

Petrova, A. V., \& Orlov, V. V. 1999, AJ, 117, 587

Press, W. H., Teukolsky, S. A., Vetterling, W. T., \& Flannery, B. P. 1992, Numerical Recipes in Fortran. The art of scientific computing, 2nd edn. (Cambridge: Cambridge University Press)

Pringle, J. E., \& Wade, R. A. 1985, in Interacting Binary Stars, eds. J. E. Pringle, \& R. A. Wade, 123

Regős, E., Bailey, V. C., \& Mardling, R. 2005, MNRAS, 358, 544

Renvoizé, V., Baraffe, I., Kolb, U., \& Ritter, H. 2002, A\&A, 389, 485

Ritter, H. 1988, A\&A, 202, 93

Savonije, G. J. 1978, A\&A, 62, 317

Sepinsky, J. F., Willems, B., \& Kalogera, V. 2007a, ApJ, 660, 1624

Sepinsky, J. F., Willems, B., Kalogera, V., \& Rasio, F. A. 2007b, ApJ, 667, 1170

Sepinsky, J. F., Willems, B., Kalogera, V.\& Rasio, F. A. 2009, ApJ, 702, 1387

Sepinsky, J. F., Willems, B., Kalogera, V., \& Rasio, F. A. 2010, ApJ, 724, 546

Siess, L. 2006, A\&A, 448, 717

Siess, L. 2007, A\&A, 476, 893

Siess, L. 2009, A\&A, 497, 463

Siess, L. 2010, A\&A, 512, A10

Siess, L., Forestini, M., \& Bertout, C. 1997, A\&A, 326, 1001

Siess, L., Dufour, E., \& Forestini, M. 2000, A\&A, 358, 593

Siess, L., Izzard, R. G., Davis, P. J., \& Deschamps, R. 2013, A\&A, 550, A100

Song, H. F., Zhong, Z., \& Lu, Y. 2009, A\&A, 504, 161

Tout, C. A., Livio, M., \& Bonnell, I. A. 1999, MNRAS, 310, 360

Ulrich, R. K., \& Burger, H. L. 1976, ApJ, 206, 509

Uryu, K., \& Eriguchi, Y. 1999, MNRAS, 303, 329

van Rensbergen, W., De Greve, J.-P., De Loore, C., \& Mennekens, N. 2008, A\&A, 487, 1129

van Rensbergen, W., De Greve, J.-P., Mennekens, N., Jansen, K., \& De Loore, C. 2011, A\&A, 528, A16

Wang, B., \& Han, Z. 2012, New Astron. Rev., 56, 122

Warner, B. 1995, in Cataclysmic Variable Stars (Cambridge: Cambridge University Press), 28

Webbink, R. F. 1976, ApJS, 32, 583

Webbink, R. F. 1977a, ApJ, 211, 486

Webbink, R. F. 1977b, ApJ, 211, 881

Willems, B., \& Kolb, U. 2004, A\&A, 419, 1057

Witt, A. N., Vijh, U. P., Hobbs, L. M., et al. 2009, ApJ, 693, 1946

Zahn, J.-P. 1977, A\&A, 57, 383 